\title{
Publishing Research Support Documents in Open Access Platform to Improve Research Impact
}

\author{
Nader Ale Ebrahim* and Yii Bonn Bong \\ ${ }^{1}$ Centre for Research Services, Institute of Management and Research Services (IPPP), University of Malaya, \\ Kuala Lumpur, Wilayah Persekutuan, 50603, Malaysia; aleebrahim@um.edu.my / yiibonn@um.edu.my \\ *Correspondence: aleebrahim@um.edu.my; Tel.: +60-3-7967-6289
}

\begin{abstract}
Researchers, journals, and universities want to receive more citations for their scholarly publications. However, a paper citations depend on its quality, visibility and author's online profile. Research support documents (unpublished papers, white papers, project reports, datasets, software, posters, online resources and teaching materials) can be additional source for increasing the author's visibility. To enhance research visibility and impact, the full range of scholarly output should be available online on the open access platform. With open access platform, key research findings are made accessible immediately to the scientific community. Therefore, the publicly available research support documents may result in receiving more citations in addition to the published papers. In this paper we conducted a simplified three stages study on the benefits of research support documents publications on open access platform. We also elaborated on approaches of improving your research visibility and impact through these document.
\end{abstract}

Keywords: Improve citations; Research tools; Research Visibility; Research impact; Documents publishing; Highly cited

\section{Introduction}

Research impact is important for researchers to improve their research reputation, increase university ranking, and getting grants from funders [1]. Research impact assessment can be done either qualitatively or quantitatively. Nevertheless, scientific outputs and activities which linked to research investments are the most favorable criteria in assessing research impact, with a major share coming from published papers. Though millions of research papers are published yearly by journals and indexed in databases such as Web of Science and Scopus, a study in 2014 reported that over $43 \%$ of Web of Science papers have never received any citations [2]. On the other hand, the most cited work might not necessarily be the best piece produced by a researcher [3]. Believing that publication is just the starting phase of research impact and visibility, the authors thus suggested proactiveness in dissemination of research publications [1]. There are more than 30 different strategies proposed to improve one's research visibility and impact [4]. One of the strategy to improve research visibility and impact is to make research support documents such as unpublished papers, white papers, project reports, datasets, software, posters, online resources and teaching materials available to all. This study will elaborate more on improving research visibility and impact through publishing research support document.

\section{Materials and Methods}

The authors conducted a simplified three stages study to investigate the effect of publishing unpublished research support document in open access platform on researcher's visibility and impact. In the first stage, the authors evaluated a simple bibliometrics analysis on the research topic on the SciVal platform. SciVal, a product developed under the Elsevier Research Intelligence, is used to visualize research performance based on SCOPUS publication, citation and usage data since 1996 [5]. SciVal enables research institutions to create an evidence based strategy. The data for this analysis 
was retrieved on 19 June 2017 through University of Malaya access point. In the second stage, the authors expanded their search for additional resources on the web for recommendations or advice to publish all scholarly outputs by university's library website. Finally, the authors added in their own experiences and ideas on improving research visibility and impact to enhance the review.

\section{Bibliometrics analysis}

SciVal offers quick, easy access to the research performance of 8,500 research institutions and 220 nations worldwide [6]. SciVal enables visualization of Elsevier's SCOPUS data for the chosen search terms "Visibility" and "Citations". Being the largest abstract and reference database, SCOPUS provides citation dataset of research literature and quality web sources [7]. Figure 1 shows the publications trend on research visibility and citations impact from 1996 to date (19 June 2017). The number of publication on the topic has risen from three publications in the year 1996 to 86 publications in 2016. In the last five years 349 papers were published, receiving over 7,650 views, 1,058 citations, 101 international collaborations, and 1.31 Field-Weighted Citation Impact (FWCI). The FWCI is a measure of citation impact that normalizes for differences in citation activity by subject field, article type, and publication year [8]. The world's average for FWCI is indexed at 1.00, as such, values above 1.00 indicate an above average citation impact. Specifically, a citation impact of 1.31 indicates $31 \%$ of the citations are above the average citations in this same filed.

The top 50 key-phrases by relevance for the past five years publications (349 publication) is shown in Figure 2. Notably, the phrases "visibility" and "impact" are among the most repeated keywords in recent publications which gained growing attentions. The trends of publications and the top 50 key-phrases have proven the importance of the research visibility and impact in scholarly publications. Therefore, there is an interest to find alternative ways to improve research visibility and impact, such as publishing research support document.

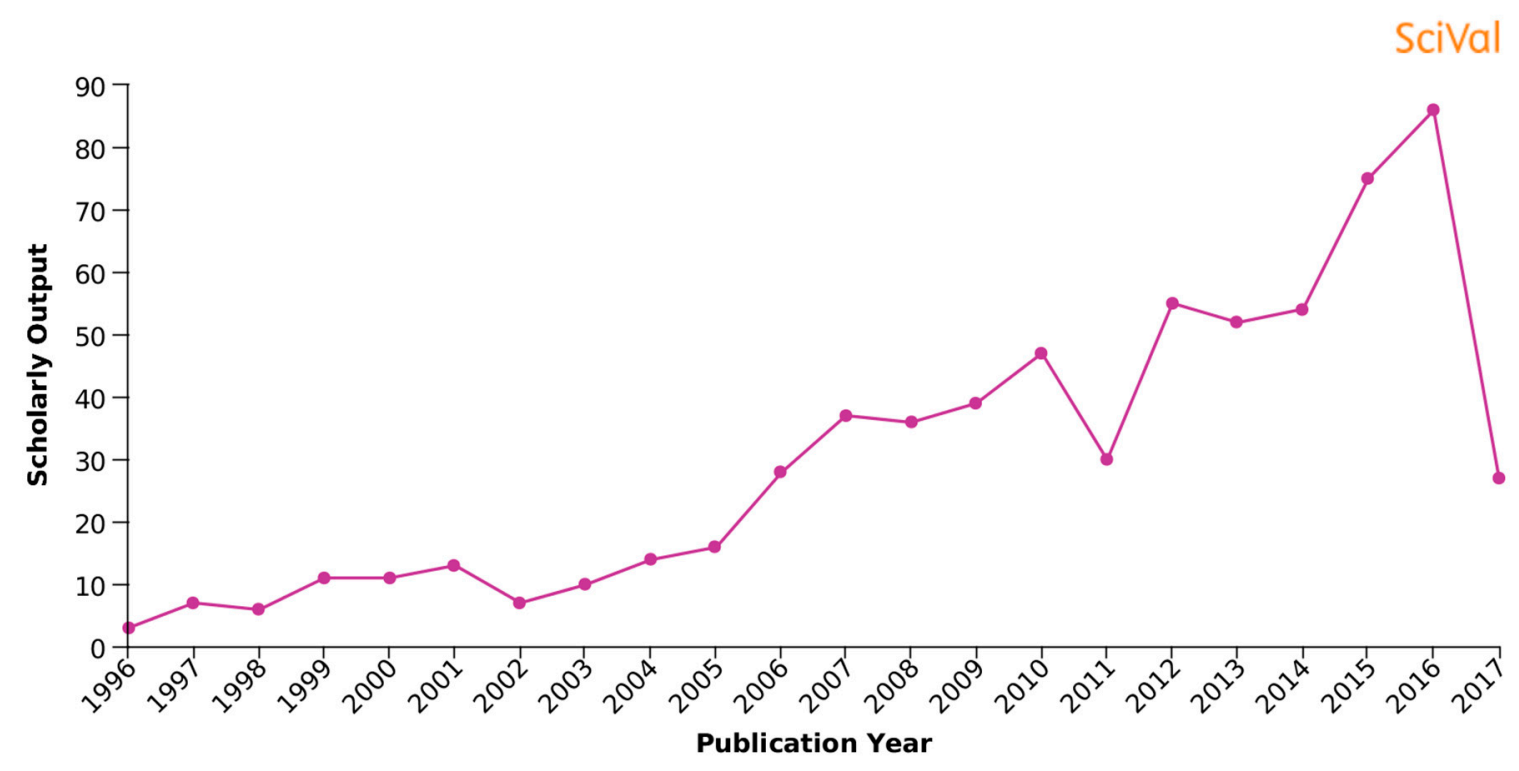

Figure 1 Trend of publications on research visibility and citations impact (data from 1996 to date) 


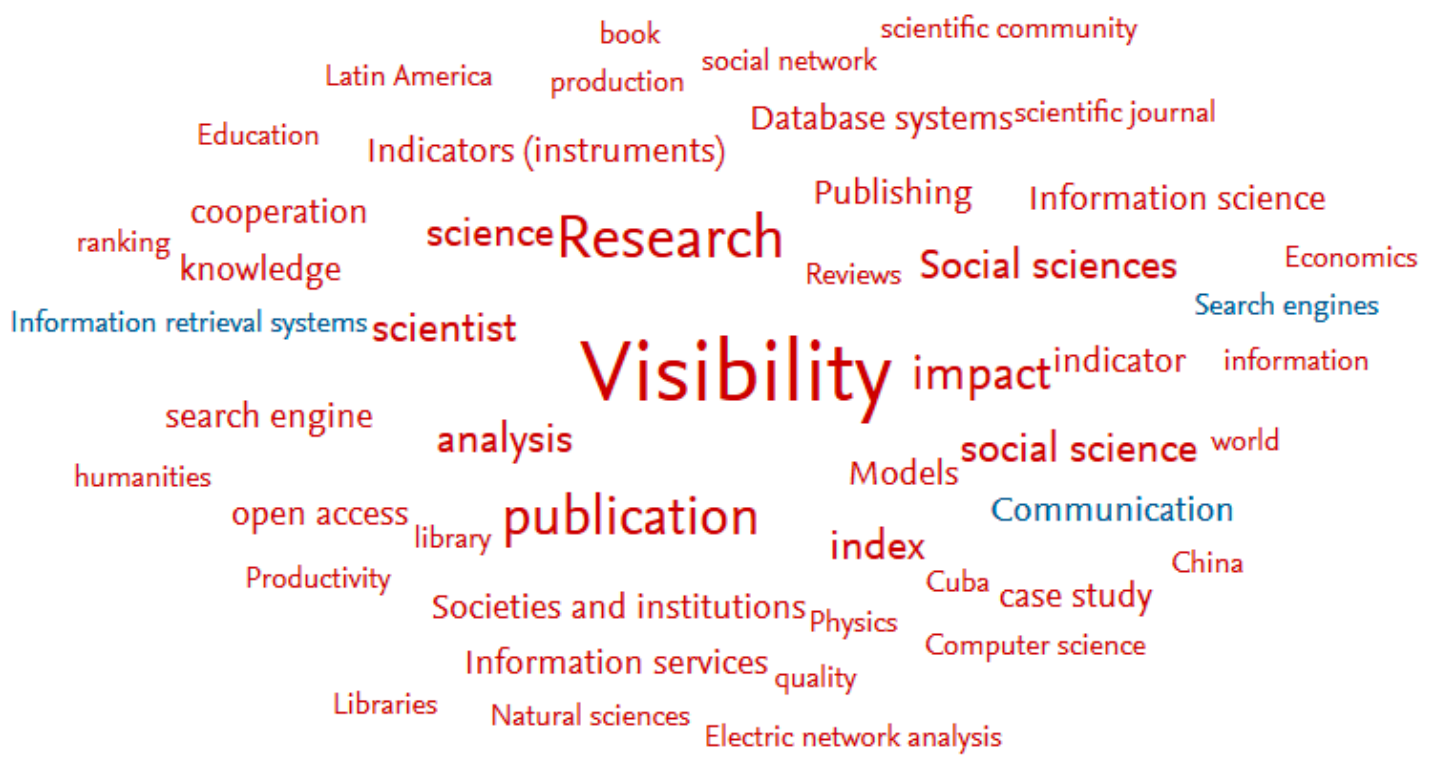

A A A relevance of keyphrase | declining $\square$ growing (2012-2016)

Figure 2 The top 50 key-phrases by relevance in the past five years papers (349 publication)

\subsection{Additional resources}

In the second stage, the authors expanded their search for additional resources on the web. Universities' library, publisher, and journal websites were explored for recommendations or advices to publish all scholarly outputs. Scholarly output defines the total count of research outputs and is used to benchmark researchers' productivity from the same disciplines and of similar career life span. The more widely accepted traditional scholarly output are printed books (including textbooks, research monographs, conference proceedings etc.), printed journal articles and other printed materials such as professional journals, newspapers and magazines [9]. The National Information Standards Organization (NISO) listed all scholarly outputs, including the traditional academic publication and extending to more alternative outputs [10]. The NISO list are available online at https://sites.google.com/a/niso.org/scholarlyoutputs/, which include: Abstract, Academic Dissertation, Annual Report, Article, Bibliography, Blog, Book Chapter, Book Review, Case report, Classical Articles, Clinical Guidelines, Clinical trial, Conference paper, Dictionary, Discussion, Editorial Material, Errata, Evaluation Study, Excerpt, Fiction and Creative Prose, Guidelines, Meeting Abstract, Meeting Summary, Meta-Analysis, Monograph, Music Score, Non-fiction Work, Observational Study, Opinion piece, Poetry, Practice Guidelines, Proceedings Paper, Script or screenplay, and TV, Radio, Film, or Theater Review. In addition, the authors would like to propose that unpublished papers, white papers, project reports, datasets, software, posters, online resources, teaching materials, non-technical documents, keynote speech, interview with newspaper, and research proposal should be made available, including published papers and "Research Support Documents".

\subsection{Authors' experiences}

The authors believe that researchers who store, preserve and disseminate their scholarly output through open access platform will receive more citations and impact. Open Access (OA) increases citation rate $[4,11]$. The changing landscape in publishing has resulted in researchers to search electronically and follow the Digital Object Identifier (DOIs) or hyperlinks rather than searching the hardcopies version. Citation rates increase even more if the scholarly output is freely accessible [4, 12]. Table 1 shows that Open Access has a positive impact on the growth of citations [4, 13]. In 
addition, the frequency of a paper being cited will also rise by having more versions of repositories in Google Scholar [14]. The researchers therefore should not only try to make their unpublished paper available, but to increase the number of Google Scholar versions as well, by depositing their scholarly outputs in open access platforms.

Table 1 Effect of Open Access (OA) on the level of citations [4, 13].

\begin{tabular}{lc}
\hline \multicolumn{1}{c}{ Discipline } & \% increase in citations with Open Access \\
\hline Physics/astronomy & 170 to 580 \\
Mathematics & 35 to 91 \\
Biology & -5 to 36 \\
Electrical engineering & 51 \\
Computer science & 157 \\
Political science & 86 \\
Philosophy & 45 \\
Medicine & 300 to 450 \\
Communication studies (IT) & 200 \\
Agricultural sciences & 200 to 600 \\
\hline
\end{tabular}

\section{Discussion}

Sharing journal articles alone is not the only way to increase the chance of getting citations. Researchers should also consider to make all scholarly outputs such as unpublished papers, white papers, project reports, datasets, software, posters, online resources, and teaching materials available online. We named all these materials as "Research Support Documents" which provide an additional opportunity to demonstrate and enhance the research work as well as to reach out to wider audiences. The "Research Support Documents" is not limited to the said items. The non-technical documents, keynote speech, interview with newspaper, and research proposal also can be categorized under "Research Support Documents". These non-technical documents lead people to search for your technical papers, and thus rise your scientific profile on the internet.

In addition, more publishers encourage the good practice of research data management, from data entry to the dissemination and archiving of valuable results. Data sharing practices have been made compulsory as part of the publication policies, especially in medical journals. Arguments and debates have been going around, claiming that publicly funded research data are public goods, hence should be disclosed to all. Besides increasing the visibility and relevance of research output, data sharing accelerates scientific progress, reduces research costs and decreases the time for results [15].

Open access platform such as Figshare (https://figshare.com/); Slideshare (https://www.slideshare.net/); Scribd (https://www.scribd.com/); SSRN (https://www.ssrn.com/); Archive (https://archive.org/); DRYD (http://datadryad.org/); ZENODO (https://zenodo.org/); and many more (you can find at part four of https://www.mindmeister.com/39583892) make it possible to open the full range of work produced by scholars and researchers to be available online. Researchers have two options for their unpublished materials, either to publish in a relevant journal or to upload in an open access repositories. Here are some journals which accept data for publications:

Scientific Data (Nature)

Biodiversity Data Journal

GeoScience Data Journal

Data in Brief (Elsevier)

Journal of Open Archaeology Data

Open Health Data

Earth System Science Data 


\section{Journal of Open Psychology}

Journal of Physical and Chemical Research Data

Journal of Open Research Software

Information sharing facilitates the advancement of science and knowledge. Data sharing, for example, benefits not just the researchers and the scientific community, but the public as well. It encourages multiple perspectives, helps to identify errors in a research, trains new researchers and avoids duplication in data collection or repetitive of similar studies [16]. In addition, data sharing also revitalizes more collaborations and connections between scientists, and leads to higher research visibility and impact. Scientists who share data publicly receive more citations [17]. This argument is also supported by a study which found that publicly available data was significantly associated with a $69 \%$ increase in citations, independently of journal impact factor, publication year and country [16].

All research has the potential for impact within and beyond academia, if it would be available online. Self-archiving is the act of authors depositing a free copy of electronic document online to provide immediate open access for the purpose of greater research visibility [18]. However, researchers are constrained by the publisher's policies when depositing in digital repository. Each publisher has written policies regarding the copyright issues and author's right to deposit a publication, and the version of publication that authors are able to deposit [19]. An empirical study has shown that although a paper may not be written and published in a high quality manner and journal, it is still possible for the paper to acquire adequate citations and world-wide recognition [20]. Once a paper visibility increases, the citation will be increased at a significant rate [21].

\section{Conclusions}

Publishing research support documents in open access platform can be a promotional source for improving the research visibility and impact. Researchers should make available all of their scholarly output such as unpublished papers, white papers, project reports, datasets, software, posters, online resources, teaching materials, non-technical documents, keynote speech, interview with newspaper, and research proposal on the open access platform for improving the research visibility and impact. Self-archiving is an alternative option to make research support documents available besides journal article publications.

\section{References}

1. Bong, Y.B. and N. Ale Ebrahim, Increasing Visibility and Enhancing Impact of Research. Asia Research News, 2017. aid/10634(cid/1): p. 1-3.

2. Van Noorden, R., B. Maher, and R. Nuzzo, The top 100 papers. Nature, 2014. 514.

3. Ioannidis, J.P., et al., Bibliometrics: Is your most cited work your best? Nature, 2014. 514(7524): p. 561-2.

4. Ale Ebrahim, N., et al., Effective Strategies for Increasing Citation Frequency. International Education Studies, 2013. 6(11): p. 93-99.

5. Blanché, S. Introducing elsevier research intelligence. 201429 September 22 June 2017]; Available from: http://ir.nrf.ac.za/bitstream/handle/10907/519/Blanche Elsevier.pdf.

6. Elsevier. About SciVal. 201719 June]; Available from: https://www.elsevier.com/solutions/scival.

7. Aghaei Chadegani, A., et al., A Comparison between Two Main Academic Literature Collections: Web of Science and Scopus Databases. Asian Social Science, 2013. 9(5): p. 18-26.

8. Jang, H. and H. Kim, Research output of science, technology and bioscience publications in Asia. Science Editing, 2014. 1(2): p. 62-70.

9. Maynard, S. and A. O'Brien, Scholarly output: print and digital - in teaching and research. Journal of Documentation, 2010. 66(3): p. 384-408.

10. Beaudoin, Y., et al., Outputs of the NISO Alternative Assessment Metrics Project. 2017, Clipper Mill Road, Suite 302, Baltimore, MD. 
11. MacCallum, C.J. and H. Parthasarathy, Open Access Increases Citation Rate. PLoS Biol, 2006. 4(5): p. e176.

12. Evans, J.A., Electronic Publication and the Narrowing of Science and Scholarship. Science, 2008. 321(5887): p. 395-399.

13. Swan, A., The Open Access citation advantage: Studies and results to date. 2010.

14. Mohammadjafari, M., et al., Relationship of Google Scholar Versions and Paper Citations. Preprint avaliable at https://www.preprints.org/manuscript/201706.0062/v1, 2017: p. 1-11.

15. Piwowar, H.A., et al., Towards a Data Sharing Culture: Recommendations for Leadership from Academic Health Centers. PLOS Medicine, 2008. 5(9): p. e183.

16. Piwowar, H.A., R.S. Day, and D.B. Fridsma, Sharing Detailed Research Data Is Associated with Increased Citation Rate. PLoS ONE, 2007. 2(3): p. e308.

17. Piwowar, H.A. and T.J. Vision, Data reuse and the open data citation advantage. PeerJ, 2013. 1: p. e175.

18. Tripathy, J., et al., Ten tips to improve the visibility and dissemination of research for policy makers and practitioners. Public Health Action, 2017. 7(1): p. 10-14.

19. Inefuku, H.W., Pre-Print, Post-Print or Offprint? A guide to publication versions, permissions and the digital repository, in Digital Repository Outreach and Workshops. 2. 2013: Digital Repository @ Iowa State University, Ames, IA.

20. Ale Ebrahim, N., et al., Visibility and Citation Impact. International Education Studies, 2014. 7(4): p. 120125.

21. Norman, E.R., Maximizing Journal Article Citation Online: Readers, Robots, and Research Visibility. Politics \& Policy, 2012. 40(1): p. 1-12. 